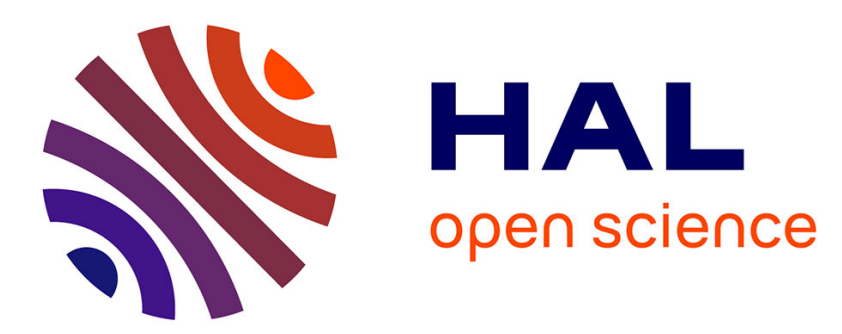

\title{
Chronologie et circulation d'individus dans le Rubané : Résultats de l'analyse des industries osseuses rubanées de l'Allemagne au Bassin parisien
}

\author{
Isabelle Sidéra
}

\section{- To cite this version:}

Isabelle Sidéra. Chronologie et circulation d'individus dans le Rubané: Résultats de l'analyse des industries osseuses rubanées de l'Allemagne au Bassin parisien. Bulletin de la Société préhistorique française, 2013, 110 (3), pp.463-472. 10.3406/bspf.2013.14291 · hal-01548587

\section{HAL Id: hal-01548587 \\ https://hal.parisnanterre.fr/hal-01548587}

Submitted on 29 Jun 2017

HAL is a multi-disciplinary open access archive for the deposit and dissemination of scientific research documents, whether they are published or not. The documents may come from teaching and research institutions in France or abroad, or from public or private research centers.
L'archive ouverte pluridisciplinaire HAL, est destinée au dépôt et à la diffusion de documents scientifiques de niveau recherche, publiés ou non, émanant des établissements d'enseignement et de recherche français ou étrangers, des laboratoires publics ou privés. 


\title{
Chronologie et circulation d'individus dans le Rubané Résultats de l'analyse des industries osseuses rubanées de l'Allemagne au Bassin parisien
}

\author{
Isabelle SIDÉRA
}

Résumé : L'étude des séries osseuses rubanées du Bade-Wurtemberg au Bassin parisien montre des ruptures et des continuités entre les échelles inter-régionale, régionale, locale ou intra-site, qui renvoient à la chronologie, mais aussi à d'autres facteurs (Sidéra, 1989, 2010 et 2012). Les caractéristiques des assemblages sont posées ici selon les trois échelles géographiques considérées. Cette confrontation permet de mettre en évidence une première rupture majeure, clairement chronologique, perceptible entre les étapes ancienne et moyenne et les étapes récente et finale du Rubané. La nature des assemblages et les pratiques techniques et fonctionnelles qui y sont associées présentent une évolution nette. En parallèle à cette évolution, une régionalisation se fait jour dès l'étape moyenne, qui s'accentue avec la fin du Rubané. La composition des assemblages comporte très tôt des signatures régionales distinctes et identifiables.

À l'échelle du Bassin parisien une seconde rupture se manifeste dans le Rubané récent du Bassin parisien (RRBP), lors de son étape finale. Cette dernière n'est pas tant caractérisée par une nouvelle évolution de nature des assemblages osseux que par l'ampleur que prennent certaines formes d'objets apparus précedemment dans le RRBP. Une continuité avec les débuts du Villeneuve-Saint-Germain est évidente.

L'évolution des assemblages osseux rubanés est significative et se manifeste donc à la fois à l'échelle large des grandes étapes de la chronologie, mais aussi à une échelle plus fine. Les industries osseuses représentent donc des indicateurs chronologiques fiables. Nous verrons cependant que la chronologie n'est pas l'unique facteur de variabilité des assemblages et ceci est un nouvel apport propre aux assemblages osseux. Ces grandes tendances évolutives ne sont en effet pas toujours sensibles à l'échelle intra-site. Il en va ainsi des bâtiments bien documentés de Cuiry-lès-Chaudardes, mais également de ceux d'autres sites mineurs. L'analyse de cette distorsion entre les échelles d'observation inter-régionale, régionale et intra-site aboutit à considérer que les assemblages osseux des bâtiments contemporains d'un même site renvoient à des traditions régionales distinctes. Ceci conduit à poser l'hypothèse d'une intégration d'individus de différentes origines ordonnée par des réseaux d'alliance matrimoniale intercommunautaires non nécessairement voisines. De nouvelles vues des mécanismes du peuplement sont ainsi posées dans cet article.

Mots-clés : Néolithique, Rubané, Bassin parisien, industrie osseuse, chronologie, réseaux d'alliance, relations exogamiques, déplacement d'individus.

\footnotetext{
Abstract: This article is based on a study of Bandkeramik (LBK) artefacts in bone and antler from sites distributed over a wide geographical area, stretching from Baden-Württemberg to the Paris basin. The study highlights breaks and continuities in trends at inter-regional, regional and local scales, reflecting not only chronology but also other factors (Sidéra 1989, 2010 and 2012). The characteristics of the assemblages in relation to these three geographical scales are described here and some new interpretations are discussed. Twenty-three types of artefact in bone, tooth and antler can be differentiated (fig. 2). A first major break is apparent at the inter-regional scale between the early/middle LBK and the late/final LBK. This is a chronological break, as there is a clear development through time in the nature of the assemblages, reflecting change in technical and functional aspects. Large perforated antler implements (fig. 2, no. 5), found on many sites from Austria to Alsace, are typical of the early and middle LBK. This type disappears in the late and final LBK. New types of artefact appear in these later stages, such as bone rings in Alsace and the Paris basin. At the same time, a process of regionalisation develops as early as the middle stage, becoming more accentuated at the end of the LBK. Thus 'T-axes' (fig. 2, type no. 3) appear in the late and final LBK of Baden-Württemberg, and continue in this region after the LBK, through the Hinkelstein, Grossgartach and Rössen sequence. Thin pointed tools with flattened epiphysis and sides (fig. 2, type no. 23; fig. 5) are found exclusively in the Paris basin.

Developments at the regional scale are best illustrated by assemblages from the Paris basin dating to the Rubané Récent $d u$ Bassin Parisien (RRBP). Here the largest corpus (456 artefacts) is from Cuiry-lès-Chaudardes and twenty-five types
} 
can be distinguished (fig. 4). Antler is used relatively little and most of the types are on bone. Edge tools show the highest morphological diversity (fig. 4, type nos. 18 to 25), followed by pointed tools (fig. 4, type nos. 9 to 13). Thin points with flattened epiphysis and sides, made on metapodials probably divided just by abrasion, become the most common type of pointed tool in the final stage of the RRBP and remain frequent in the early stage of Villeneuve-Saint-Germain (fig. 6). A clear continuity can thus be seen between the latest LBK and the beginnings of Villeneuve-Saint-Germain, as has also been shown for pottery and the flint industries (Constantin and Ilett, 1997; Allard, 2005). All this evidence demonstrates the existence of chronological trends in LBK bone industries, not only over a broad temporal range but also over shorter intervals of time. Bone artefacts can indeed be quite reliable chronological indicators.

At the local or intra-site scale, chronology is apparently not the only factor contibuting to assemblage variability. This can be illustrated by examing the distribution of rings and thin points with flattened epiphysis and sides through the sequence of three ceramic phases established for Cuiry-lès-Chaudardes. In the earliest phase, there are very few rings and flat-sided points. In the middle phase, the flat-sided points are present in all the house contexts, excepting house no. 440 and possibly also house no. 380. Rings are more common as well, occurring notably with house no. 440 and no. 380. In the last phase, flat-sided points are more frequent in all the contexts. Thus the bone tool assemblages from houses no. 440 and perhaps also no. 380 are anomalous in terms of chronology. While the chronological factor undeniably contributes to assemblage variation on the site, this does not apply to all the houses. Chronology cannot explain the absence of flat-sided points in certain house contexts. Another explanation must therefore be found.

The hypothesis put forward here is that the bone artefact assemblages from contemporary house contexts on the settlement result from different regional traditions. Thus the assemblages with bone rings but without flat-sided points could reflect a tradition from upper Alsace and Baden-Württemberg. The assemblages associating rings and flat-sided points could reflect a mixture of LBK and Cardial technological traditions (Sidéra, 2008, 2010 et 2012; Sidéra et al., 2010). Very similar thin flat-sided points occur in the Cardial/Epicardial of Spain (Bosch et al., 2000, fig. 199), as do bone rings in graves (Yravedra Sainz de los Terreros et al., 2008). The mixed tradition could have come to the Aisne valley via the Champagne region, from where much of the flint at Cuiry-lès-Chaudardes originates (Allard, 2005). The presence of these distinct traditions within the site can thus be seen as evidence for networks of movement of people within the LBK, probably based on kinship and exogamy (fig. 7). This offers a new view of LBK society, involving highly mobile individuals and quite permeable social groups, and resulting in diversity in the population structure of settlements.

Keywords: Neolithic, Bandkeramik, Paris basin, bone artefacts, chronology, alliance networks, exogamic relations, movement of individuals.

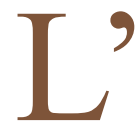

INDUSTRIE OSSEUSE rubanée est un document étudié depuis une quarantaine d'années seulement. Les premières publications solides sur la question - autrement dit, qui isolent les objets et tentent de les traiter en tant qu'ensemble - prennent place à la fin des années 1970 et surtout dans les années 1980 (Poplin, 1975, 1976 et 1980; Poulain, 1983; Mathieu, 1983; Rulf, 1984; Gallay et Mathieu, 1988; Sidéra, 1989; Kaufmann 1989). Sauf exception, ces publications concernent quelques pièces marquantes au sein des assemblages, rarement des ensembles complets. Afin de documenter la question et de dresser un portrait d'ensemble pertinent, il a donc fallu rassembler le corpus et étudier les séries à un niveau d'ensemble, autant du point de vue des formes, que des techniques et des fonctions; à mon sens, l'unique façon de parvenir à l'intention via la compréhension du système de production.

Dans les années 1990, j'ai commencé à caractériser les assemblages rubanés des grandes régions où la matière osseuse était conservée : du Bade-Wurtemberg au Bassin parisien. Dans le Bade-Wurtemberg comme en Alsace, les assemblages osseux sont associés à toutes les étapes du Rubané, depuis le Flomborn (Rubané ancien) jusqu'à la fin du Rubané (Sidéra, 1993 et 2000). Dans le Bassin parisien, ce sont les dernières étapes qui sont représentées, par le Rubané récent du Bassin parisien (RRBP). Signalons qu'il n'y a pas d'objets en matière osseuse dans le Rubané moyen champenois ou alors ils sont peu nombreux et inédits.
Tenant compte des publications, en intégrant la Bavière, l'Allemagne orientale, l'Autriche et la Moravie, les séries d'une cinquantaine de sites peuvent être considérées au total (fig. 1). Celles-ci permettent d'établir la première typologie de l'industrie osseuse du Rubané et de mettre au jour des ruptures et des continuités (Sidéra, 1989, 2008 et 2010). L'objet de cet article est de présenter des hypothèses explicatives de la formation et de la variation des compositions des assemblages osseux rubanés aux échelles inter-régionale, régionale, locale et intra-site.

\section{ÉVOLUTION CHRONOLOGIQUE ET RÉGIONALISATION : DU BADE-WURTEMBERG AU BASSIN PARISIEN}

$\mathrm{L}$ es principaux types représentés dans les assemblages de trois grandes régions, Neckar, Main, Rhin supérieur, Alsace et Bassin parisien, ont été caractérisés pour mesurer la cohérence des assemblages. Vingt-trois types d'objets en os, dents et bois de cerf, dans le détail desquels nous n'entrerons pas ici ont été différenciés (fig. 2) ${ }^{1}$. Un inventaire précis de ces collections a été publié en 2000 (Sidéra, 2000). Il convient de lui ajouter cinq nouvelles séries bien documentées dont deux étudiées par l'auteur : Vaihingen (environ 180 pièces : Sidéra, 1998), Cuiry- 


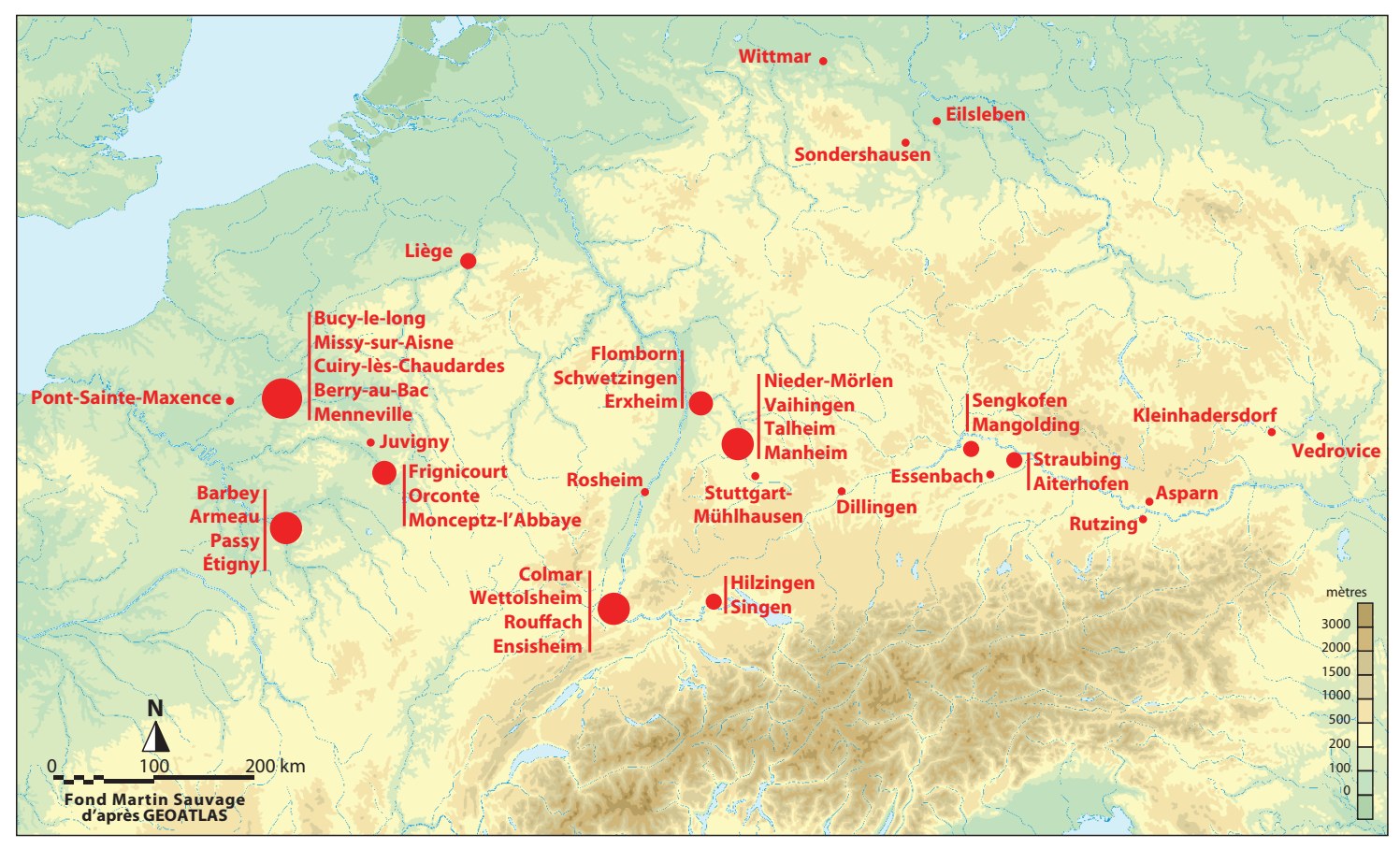

Fig. 1 - Situation des principaux sites d'habitat ou funéraires du Rubané avec industrie osseuse publiée.

Fig. 1 - Distribution of main settlement or funerary sites with published bone industries.

lès-Chaudardes (456 pièces : Sidéra, 2010), Herxheim (448 objets : Haack, 2012) Bad Nauheim-Nieder-Mörlen (plus de 200 objets : Huser 2002) et Asparn (150 objets environ : Fehlmann 2010).

Sur la typologie proposée plusieurs commentaires peuvent être formulés :

1) Deux macro-ensembles peuvent être distingués : l'un avec une présence forte du bois de cerf, dont de grandes masses perforées pour être emmanchées (fig. 2, types $n^{\text {os }} 1$ à 10), l'autre avec de plus petits objets principalement élaborés en os, secondairement en dents et en bois de cerf (fig. 2, types ${ }^{\text {os }} 11$ à 23). Ces ensembles scindent l'espace géographique considéré, avec un groupe Est Rhin supérieur/Main/Neckar/Alsace (fig. 2, types $n^{\text {os }} 1$ à 4 ) et un groupe Ouest Bassin parisien (fig. 2, type $n^{\circ} 23$ ).

2) Des spécificités partagées entre deux régions voisines se font jour : entre l'Alsace et le Bade-Wurtemberg (fig. 2, types $\mathrm{n}^{\text {os }} 5$ à 10 ) et entre l'Alsace et le Bassin parisien (fig. 2, types $n^{\text {os }} 20$ et 21 ).

3) Un lot d'objets commun aux trois régions considérées apparaît : il forme le socle identitaire du Rubané (fig. 2, types nos 11 à 19).

Ces spécificités géographiques sont chronologiques. Ainsi, les grosses masses perforées pour l'emmanchement, présentes dans de nombreux sites depuis l'Autriche jusqu'à l'Alsace, sont typiques du Rubané ancien et moyen (fig. 2, type $n^{\circ} 5$; Hüser, 2002; Fehlmann, 2010 ; Sidéra, 1998 et 2000; Ruck, 2001). La représentation de ces objets s'accompagne, en outre, d'une exploitation du bois de cerf bien supérieure à ce qu'elle sera plus tard à la fin du Rubané. À titre d'exemple, 25\% d'objets façonnés dans cette matière sont comptés sur un échantillon (131 pièces) de la série de Vaihingen (Sidéra, 1998). Dans la période suivante, en Alsace et en Bassin parisien, l'exploitation du bois de cerf tombe nettement (Sidéra, 1993 et 2000). À titre d'exemple, les objets en bois de cerf du site très représentatif de Cuiry-lès-Chaudardes représentent un peu plus de $3 \%$ du corpus des objets. Les gros instruments typiques du Rubané ancien et moyen ne sont plus représentés ni dans le Rubané récent et final d'Alsace ni dans celui de la région du Rhin supérieur/Main/ Neckar. Ils sont également absents du RRBP.

En revanche, l'os en tant que matière et de nouvelles formes d'objets s'imposent dans les séries du Rubané récent et final d'Alsace comme dans celles du RRBP. Nous pouvons citer les anneaux, pièces communes au Rubané final d'Alsace et au RRBP. Quant aux outils pointus à flancs et épiphyse aplatis et peu épais, ils sont présents dans tous les sites rubanés du Bassin parisien (Sidéra, 2010). Ces données dévoilent un assemblage osseux en forte évolution à l'intérieur des étapes du Rubané, évolution étroitement liée à celle des activités économiques et fonctionnelles pratiquées dans les sites ainsi qu'à des jeux d'interactions entre sites ou régions.

En parallèle aux évolutions chronologiques du matériel, des accents régionaux de la production osseuse commencent à se faire jour dans les étapes ancienne et moyenne du Rubané. Ainsi, les objets coudés en os ou en coquille sont spécifiques au Bade-Wurtemberg où on les rencontre à Vaihingen et dans une sépulture de Flomborn (fig. 2, type ${ }^{\circ} 4$; Sidéra, 1998 et 2010). La régionalisation devient encore plus apparente dans les étapes récente et 


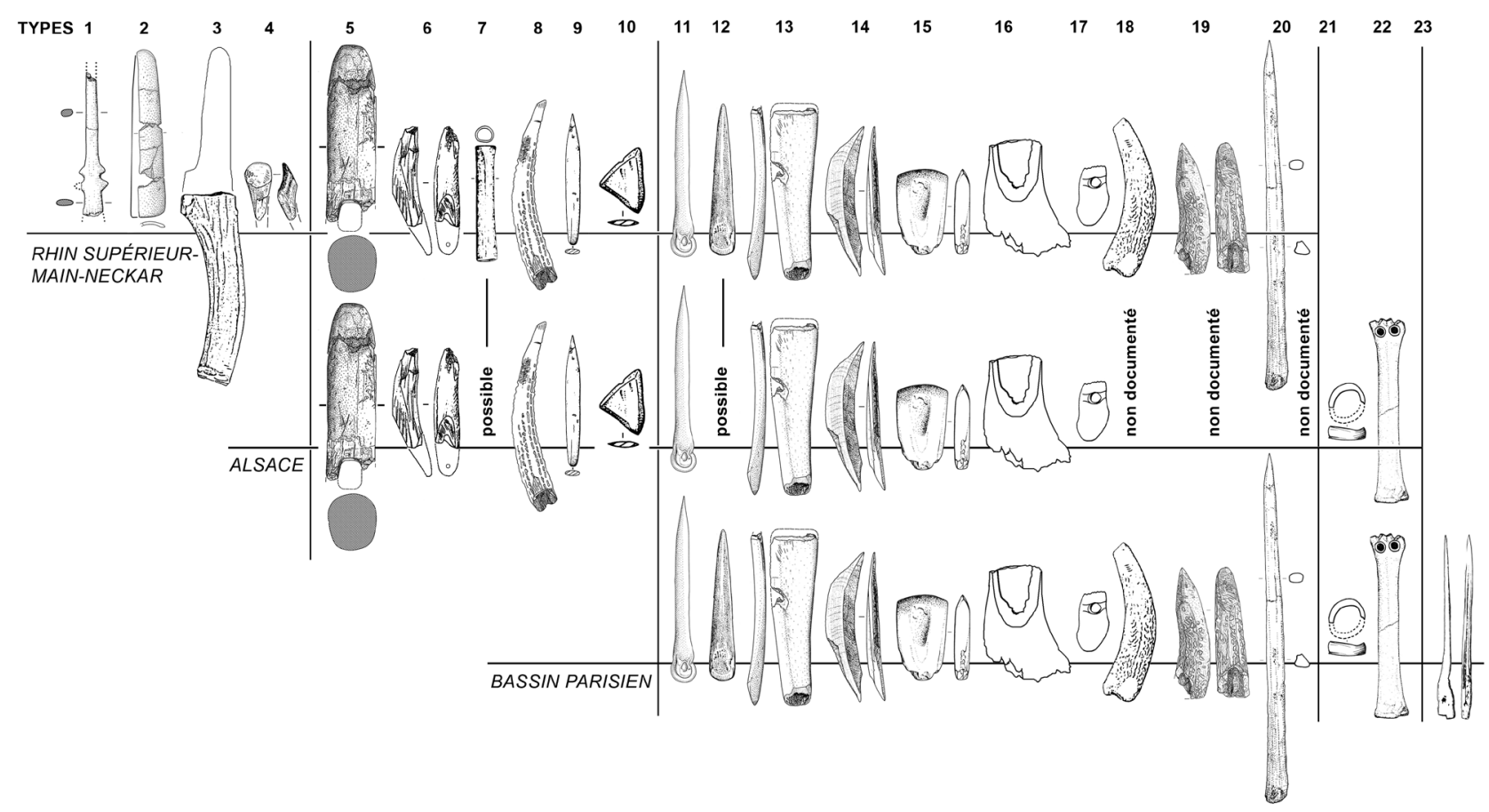

Fig. 2 - Typologie des industries rubanées du Bade-Wurtemberg au Bassin parisien. 1 : figurine anthropomorphe plate (os); 2 : outil sur longue et large baguette (os ou bois de cerf); 3 : hache en « $T$ » (bois de cerf); $4:$ objet coudé (os); $5:$ masse longue et lisse à terminaison pointue ou diffuse et perforation centrale (bois de cerf); $6:$ andouiller à base biseautée et perforée ou non (bois de cerf); 7 : tube (os); 8 : outil à plage sur andouiller (bois de cerf); $9:$ outil à plage et à pointe sur baguette (os); $10:$ : armature de flèche » triangulaire $(\mathrm{os}) ; 11$ : outil pointu débité en deux sur métapode de ruminant à terminaison non modifiée; 12 : outil pointu débité en deux sur métapode de petit ruminant à terminaison modifiée; 13 : grattoir sur côte entière ou fendue au tranchant droit ou convexe; 14 : racloir sur lame de canine de porc ou sanglier; $15:$ herminette ou hache en os; $16:$ herminette fruste (os); $17:$ crache de cerf perforée; 18 : outil pointu sur andouiller (bois de cerf); 19 : outil tranchant sur andouiller (bois de cerf); 20 : outil pointu sur métapode de ruminant scié en quart; 21 : anneau scié transversalement (os); 22 : figurine anthropomorphe sur métapode de ruminant entier avec pastillage ou incrustation de nacre; 23 : outil pointu peu épais sur métapode distal de ruminant à flancs aplatis.

Fig. 2 - Typology of Bandkeramik industries from Baden-Württemberg to the Paris basin. 1: flat anthropomorphic figurine (bone); 2: tool on long wide rod (bone or antler); 3: 'T' axe (antler); 4: bent object (bone); 5: long smooth hammer with pointed or diffuse end and central perforation (antler); 6: tine with bevelled base, perforated or not (antler); 7: tube (bone); 8: tool with flat zone on tine (antler); 9: tool with flat zone and point on rod (bone); 10: triangular "arrowhead" (bone); 11: pointed tool on ruminant metapodial split in two with unmodified end; 12: pointed tool on ruminant metapodial split in two with modified end; 13: scraper on whole or split rib with straight or convex working edge; 14: side-scraper on pig or wild boar canine; 15: bone adze or axe; 16: rough adze (bone); 17: perforated red deer canine; 18: pointed tool on tine (antler); 19: edge tool on tine (antler); 20: pointed tool on ruminant metapodial sawn in quarter; 21: transversely sawn ring (bone); 22: anthropomorphic figurine on whole ruminant metapodial with applied or incrusted mother of pearl; 23: thin pointed tool with flattened sides on ruminant distal metapodial.

finale, où certains types d'objet sont d'une diffusion géographique limitée (fig. 3). Il en va ainsi des « haches en $\mathrm{T}$ » (fig. 2, type $\mathrm{n}^{\circ}$ 3) du Rubané récent et final du BadeWurtemberg (Sidéra, 1998 et 2010). Cette forme d'objet continue de se développer dans cette même région et audelà, dans le Hinkelstein (Fritsch, 1998), le Grossgartach (Schlitz, 1901) et le Roessen (Dümmer : Werning, 1983; Heidelberg : Spatz, 1988).

De même, si les andouillers à base abrasée et ou non perforée ont un très large espace de diffusion (fig. 2, type $\mathrm{n}^{\circ} 5$ ), du Neckar à l'Alsace et de la Bavière à l'Autriche, celui-ci ne s'étend pas jusqu'au Bassin parisien (Sidéra, 2010). Dans cette région, il n'existe qu'un seul exemplaire isolé à Cuiry-lès-Chaudardes et d'une forte dérive morphologique (fig. 4, no 5).

Différents instruments en os ont une répartition régionale différente et plus circonscrite que celle des objets en bois de cerf. Ainsi, les anneaux et les figurines en volume réunissent le Bassin parisien et la haute Alsace (Jeunesse, 1995; Sidéra, 2000). Les outils pointus peu épais à épiphyse et flancs plats (fig. 2, type $\mathrm{n}^{\circ} 23$ ) sont exclusifs dans le Bassin parisien : Aisne, Oise, Champagne, Yonne et Seine-et-Marne (fig. 3).

Ces répartitions chrono-géographiques des types d'objets en matière osseuse dévoilent des réseaux de diffusion et d'échange entre les différentes entités micro-régionales rubanées. Ces réseaux sont diversifiés et changeants au cours du temps. Ainsi, la variabilité chrono-géographique du répertoire des objets en matière osseuse rubanés décrit un monde en évolution sur le plan diachronique et en interconnexion sur le plan synchronique (Sidéra, 2008, 2010 et 2012; Sidéra et al. 2010). 


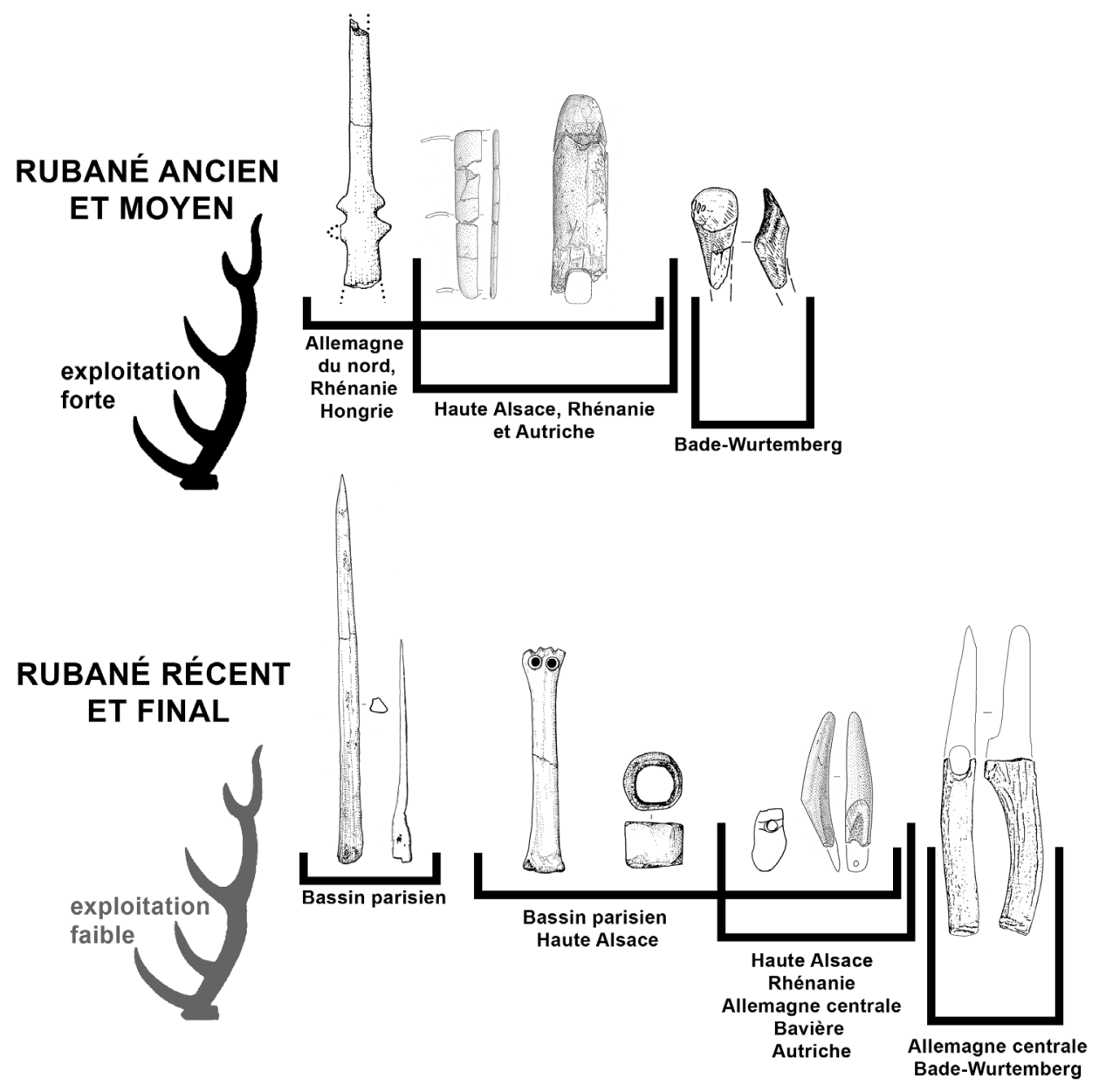

Fig. 3 - Évolution chronologique des industries osseuses du Rubané, entre Bade-Wurtemberg et Bassin parisien.

Fig. 3 - Chronological development of Bandkeramik bone industries, from Baden-Württemberg to the Paris basin.

\section{CHRONOLOGIE FINE DANS LE BASSIN PARISIEN}

$\mathrm{R}$ esserrons maintenant la chronologie et l'espace considéré et focalisons-nous sur le RRBP. Nous nous appuierons sur les vingt-cinq types d'objet issus de l'analyse du corpus de Cuiry-lès-Chaudardes (456 pièces), le corpus le plus complet au niveau régional (fig. 4).

Comme nous l'avons déjà évoqué, l'os est valorisé pour la réalisation d'objets d'une diversité morphologique importante. Les outils tranchants ont les formes les plus diversifiées, constituant de nombreux types (fig. 4, types $\mathrm{n}^{\text {os }} 18$ à 25 ). La variété typologique des outils pointus est elle aussi importante, bien qu'un peu moins diversifiée que les outils tranchants (fig. 4, types nos 9 à 13).

Parmi les éléments rares ou remarquables en os, citons un peigne à deux dents probablement employé pour décorer la céramique (fig. 4 , type $n^{\circ} 14$ ), de possibles petits manches sur phalange de bovinés (fig. 4, type $n^{\circ} 15$ ), une probable figurine sur phalange de capriné (fig. 4, type $n^{\circ} 16$ ) et des anneaux (fig. 4 , type $n^{\circ} 17$ ). Les objets en bois de cerf sont peu nombreux, peu diversifiés et principalement fabriqués à partir d'andouillers (fig. 4 , types ${ }^{\text {os }} 5$ à 8 ).
Les dents comprennent surtout des racloirs sur défenses de sanglier (fig. 4 , types $n^{\text {os }} 1$ et 2 ), de curieux objets en cylindre utilisés en percussion, le plus souvent élaborés sur des molaires de bovinés (fig. 4 , type $\mathrm{n}^{\circ} 3$ ) et de la parure sur dents, ici sur canine d'ours (fig. 4, type $n^{\circ} 4$ ).

Entre le RRBP et son étape finale aucune différence typologique qualitative n'apparaît clairement. En revanche, un développement de la forme des outils pointus peu épais à épiphyse et flancs plats et fort probablement débités uniquement par abrasion (fig. 5) se produit à l'échelle régionale, au détriment des autres types d'outil pointu, notamment le type le plus classique et le plus commun dans le Rubané : sur métapode de petit ruminant scié en deux. Ce type d'outil peut présenter parfois une abrasion superficielle des faces épiphysaires (fig. 4, types $\mathrm{n}^{\mathrm{os}} 11$ et 12 ). En tout cas, la proportion de ces outils pointus plats et peu épais devient significative du point de vue chronologique et caractérise une continuité régionale, manifeste jusque dans l'étape ancienne du VilleneuveSaint-Germain (fig. 6; Sidéra, 2008 et 2010). Ainsi, certains assemblages de maisons de la fin du Rubané, tels ceux des maisons $\mathrm{n}^{\circ} 225$ de Cuiry-lès-Chaudardes et $\mathrm{n}^{\circ} 20$ de Bucy-le-Long « la Fosselle » sont-ils marqués par un très fort taux de ces objets. 


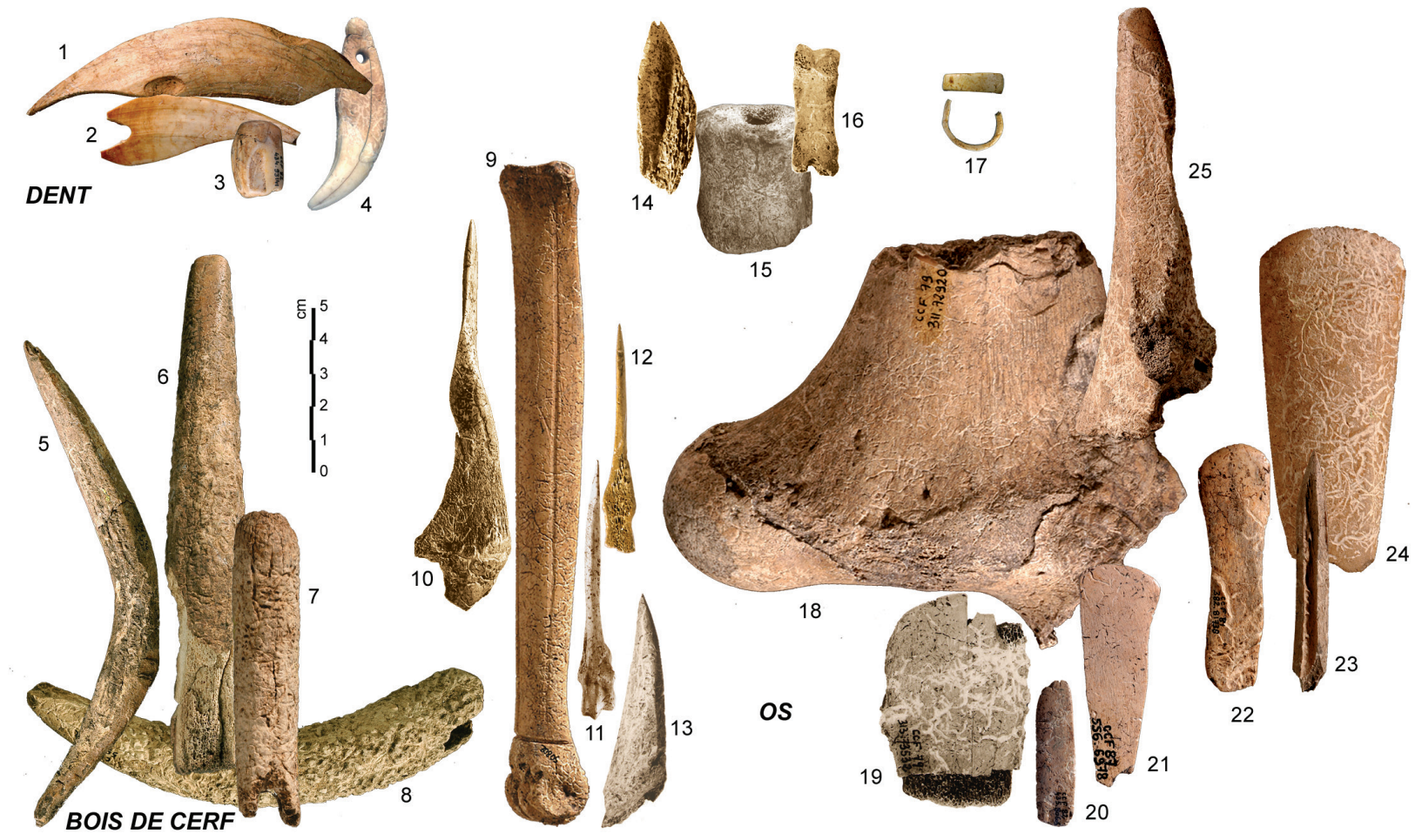

Fig. 4 - Présentation des types de l'industrie osseuse de Cuiry-lès-Chaudardes. 1 : racloir sur lame de canine de suidé; $2:$ racloir sur lame de canine de suidé perforée; 3 : élément de masse sur molaire de boviné; 4 : canine percée d'ours; $5:$ andouiller à base biseautée et perforée ou non; $6:$ outil tranchant sur andouiller; $7:$ manche perforé sur andouiller; $8:$ outil pointu sur andouiller probablement emmanché; 9 : outil pointu sur ulna; 10 : outil pointu sur métapode proximal de grand ruminant scié en quart; 11 : outil pointu sur métapode de petit ruminant scié en deux aux flancs et épiphyse non transformés ou peu; 12 : outil pointu peu épais sur métapode distal de petit ruminant à flancs aplatis; 13 : outil pointu sur éclat d'os indifférencié; 14 : peigne en os à deux dents; $15:$ phalange de bœuf perforée; 16 : possible figurine sur phalange de capriné; 17 : anneau; 18 : outil tranchant sur tronçon transversal d'os long (herminette fruste); 19 : outil tranchant sur tronçon transversal de côte de grand ruminant, à taillant droit ou convexe et lisse; 20 : outil tranchant sur tronçon transversal de côte de grand ruminant, à taillant droit ou convexe cranté; 21 : outil tranchant sur côte fendue de grand ruminant, à taillant droit ou convexe; 22 : outil tranchant sur éclat d'os long indifférencié à taillant large et facial, droit ou convexe; 23 : outil tranchant sur éclat d'os indifférencié à taillant axial étroit; 24 : forme de bottier en os ; 25 : outil tranchant sur ulna de grand ruminant.

Fig. 4 - Presentation of types for the bone industry of Cuiry-lès-Chaudardes; 1: side-scraper on suid canine; 2: side-scraper on perforated suid canine; 3: hammer component on bovine molar; 4: perforated bear canine; 5: tine with bevelled base, perforated or not; 6: edge tool on tine; 7: perforated haft on tine; 8: pointed tool on tine, probably hafted; 9: pointed tool on ulna; 10: pointed tool on large ruminant proximal metapodial sawn in quarter; 11: pointed tool on small ruminant metapodial sawn in two with unmodified or barely modified sides and epiphysis; 12: thin pointed tool on small ruminant distal metapodial with flattened sides; 13: pointed tool on undifferentiated bone flake; 14: bone comb with two teeth; 15: perforated cattle phalanx; 16: possible figurine on caprine phalanx; 17: ring; 18: edge tool on transverse section of long bone (rough adze); 19: edge tool on transverse section of large ruminant rib, with straight or convex and smooth edge; 20: edge tool on transverse section of large ruminant rib, with notched straight or convex edge; 21: edge tool on split rib of large ruminant, with straight or convex edge; 22: edge tool on undifferentiated long bone flake, with wide edge, straight or convex; 23: edge tool on undifferentiated bone flake, with narrow axial edge; 24: bone adze; 25: edge tool on large ruminant ulna.

Ces résultats sur la chronologie fine du RRBP ne sont pas spécifiques aux industries osseuses. Ils font échos aux évolutions mises en évidence sur la céramique et l'industrie lithique (Constantin et Ilett, 1997; Allard, 2005).

\section{COMPOSITION DES ASSEMBLAGES À L'ÉCHELLE DES BÂTIMENTS DE CUIRY-LÈS-CHAUDARDES}

E xaminons maintenant l'échelle intra-site et considérons la répartition par bâtiments des éléments chronologiquement significatifs tels les outils pointus peu épais à épiphyse et flancs plats et les anneaux en os, selon le phasage chronologique établi par M. Ilett sur la base de la céramique décorée. Dans la phase la plus ancienne, les outils pointus sur métapode de petit ruminant peu épais à flancs et épiphyses aplatis et les anneaux sont rares dans les maisons. Ils sont représentés à l'unité dans quelques rares bâtiments. Dans la phase moyenne, les outils pointus peu épais à flancs plats sont toujours présents à l'unité, mais leur présence est systématique dans les assemblages bien documentés, à l'exception d'une maison ( $\left.\mathrm{n}^{\circ} 440\right)$ et peut-être d'une deuxième ( $\left.\mathrm{n}^{\circ} 380\right)$. Quant aux anneaux, ils sont représentés dans plusieurs bâtiments, dont ces deux maisons n ${ }^{\text {os }} 440$ et 380 . Enfin, dans la phase récente, les outils à flancs plats sont systématiques et présents 

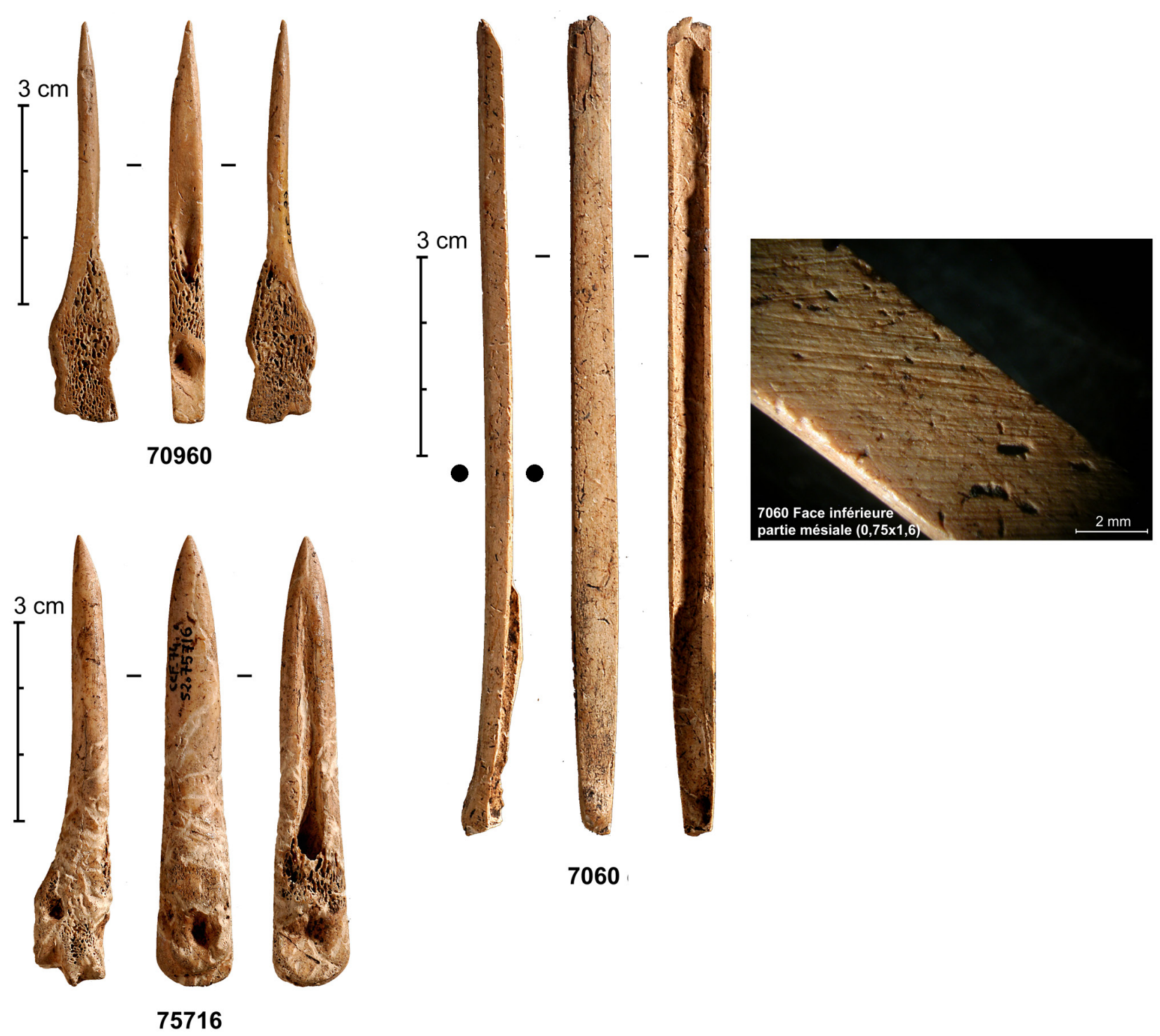

Fig. 5 - № 70960 : outil pointu peu épais sur métapode à épiphyse et flancs plats obtenu par abrasion intégrale; no 7060 : ébauche d'un outil pointu peu épais à épiphyse et flancs plats obtenu par abrasion intégrale; vue macroscopique de traces d'abrasion liées au débitage, présentes sur le corps de l'outil, photographie prise où marqué par des points sur la pièce; $\mathrm{n}^{\circ} 75716$ : outil pointu scié en deux avec aménagement de l'épiphyse par abrasion (n ${ }^{\text {os }} 70960,7060,75716$ : clichés S. Oboukhoff, MAE, Nanterre; vue macroscopique : auteur).

Fig. 5 - No. 70960: thin pointed tool on metapodial with epiphysis and sides completely flattened by abrasion; no. 7060: rough-out of a thin pointed tool on metapodial with epiphysis and sides completely flattened by abrasion; macroscopic image of traces of abrasion linked to debitage, dots indicate the position of the photograph on the artefact; no. 75716: pointed tool on metapodial sawn in two, with epiphysis modified by abrasion (nos. 70960, 7060, 75716: photos S. Oboukhoff, MAE, Nanterre; macroscopic photograph: author).

à plus d'un exemplaire dans tous les assemblages bien documentés. Les anneaux sont toujours représentés dans plusieurs bâtiments. L'assemblage du bâtiment $\mathrm{n}^{\circ} 225$, parmi les plus récents du site (Constantin et Ilett, 1997), rassemble plus de la moitié de l'effectif total des objets de ce type : produits finis et matrices de fabrication. Les assemblages d'outils pointus des bâtiments no 440 sûrement, et $\mathrm{n}^{\circ} 380$ peut-être, constituent donc des anomalies du point de vue chronologique (Sidéra, 2010). À l'échelle intra-site, le facteur chronologique n'est donc pas entièrement applicable. Un facteur chronologique joue indéniablement sur la composition des assemblages, mais sans qu'il puisse s'appliquer à tous les assemblages des maisons du site. En effet, il ne permet pas d'expliciter l'absence d'outil pointu peu épais à épiphyse et flancs plats de certains bâtiments. Un autre paradigme doit donc être adopté pour expliciter cette absence.

\section{L'HYPOTHÈSE NOUVELLE DE RÉSEAUX D'ALLIANCES MATRIMONIALES}

T a composition des assemblages « anneau, pas d'outil

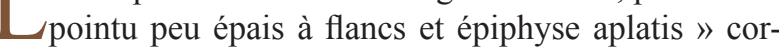
respond au Rubané final de haute Alsace et du BadeWurtemberg (fig. 2). Il n'est donc pas impensable de prêter à cette composition une origine de ces régions. 


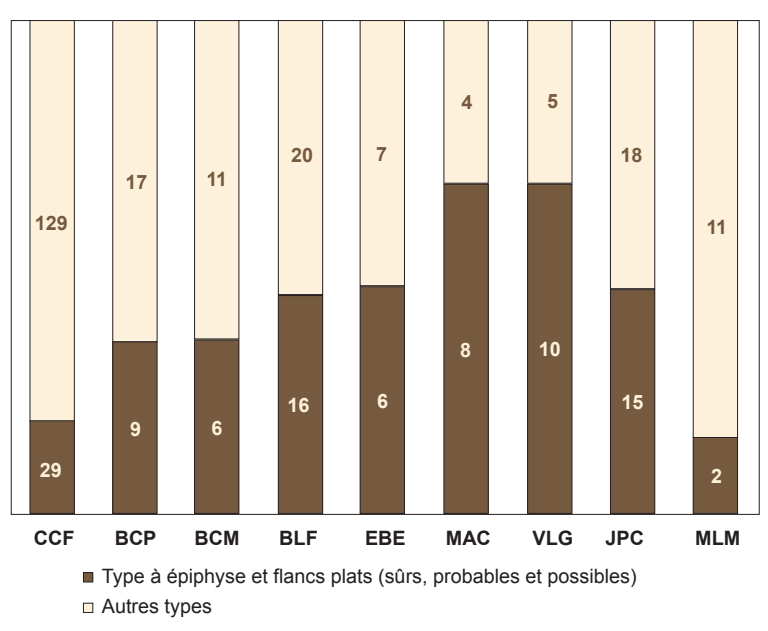

Fig. 6 - Proportion des outils pointus à flancs plats par rapport aux autres types d'outils pointus dans les sites rubanés et Villeneuve-Saint-Germain du Bassin parisien (d'après Sidéra 2010). Sites rubanés : Cuiry-lès-Chaudardes (CCF), Berry-au-Bac « le Chemin de la Pêcherie » (BCP), Berry-au-Bac « la Croix Maigret » $(\mathrm{BCM})$, Bucy-le-Long « la Fosselle » (BLF), Étigny, « le Brassot-Est » (EBE), Missy-sur-Aisne « le Culot» (MAC). Sites Villeneuve-Saint-Germain : Villeneuve-la-Guyard (VLG), Jablines « la Pente de Croupeton » (JPC), Mareuil-les-Meaux (MLM).

Fig. 6 - Proportion of flat-sided pointed tools (shaded) in relation to other types of pointed tool in Bandkeramik and Villeneuve-Saint-Germain sites in the Paris basin (after Sidé$r a, 2010)$. Bandkeramik sites : Cuiry-lès-Chaudardes (CCF), Berry-au-Bac 'le Chemin de la Pêcherie' (BCP), Berry-au-Bac 'la Croix Maigret' (BCM), Bucy-le-Long 'la Fosselle' (BLF), Étigny 'le Brassot-Est' (EBE), Missy-sur-Aisne 'le Culot' (MAC). Villeneuve-Saint-Germain sites : Villeneuve-la-Guyard (VLG), Jablines 'la Pente de Croupeton' (JPC), Mareuil-lèsMeaux (MLM).
La composition « anneau, outil pointu peu épais à flancs plats », quant à elle, renvoie à mon sens à une autre origine géographique. Elle évoque un mélange entre la tradition technique rubanée et la tradition cardiale - Cardial ou Épi-Cardial (Sidéra, 2008, 2010 et 2012; Sidéra et al., 2010). Précisons que le type outil pointu sur métapode de petit ruminant peu épais à flancs plats préexiste à la Draga, dans le contexte du Cardial ibère (Bosch et $a l .$, 2000, fig. 199). En outre, les techniques de fabrication et la morphologie des pièces de la Draga sont extrêmement similaires à certains exemplaires de Cuiry-lèsChaudardes (Sidéra, 2008; Sidéra et al., 2010). Signalons également une sépulture du Cardial ibère, dont le défunt est paré d'un anneau en os au doigt (Yravedra Sainz de los Terreros et al., 2008). La Champagne, où ces deux types d'objet existent en contexte funéraire (Sidéra, 2000), d'où provient également une très grosse partie des matières premières lithiques (Allard, 2005), pourrait assurer le relais des traditions méridionales et constituer la zone d'origine de cette composition.

L'hypothèse que je propose est donc de prêter un caractère identitaire à ces traditions de fabrication des objets en os différenciées par bâtiments, et de les rattacher à la partie visible d'un réseau de circulation de personnes issues de différentes communautés à l'intérieur du Rubané. Via l'industrie osseuse, deux réseaux de circulation croisés pourraient être détectés. Un premier réseau oriental, peutêtre lié à la haute Alsace, et un second, méridional lié,

Fig. 7 - Deux réseaux d'alliance dans le Rubané récent du Bassin parisien (DAO d'après G. Monthel).

Fig. 7 - Two alliance networks in the Paris basin final Bandkeramik RRBP (CAD after G. Monthel).

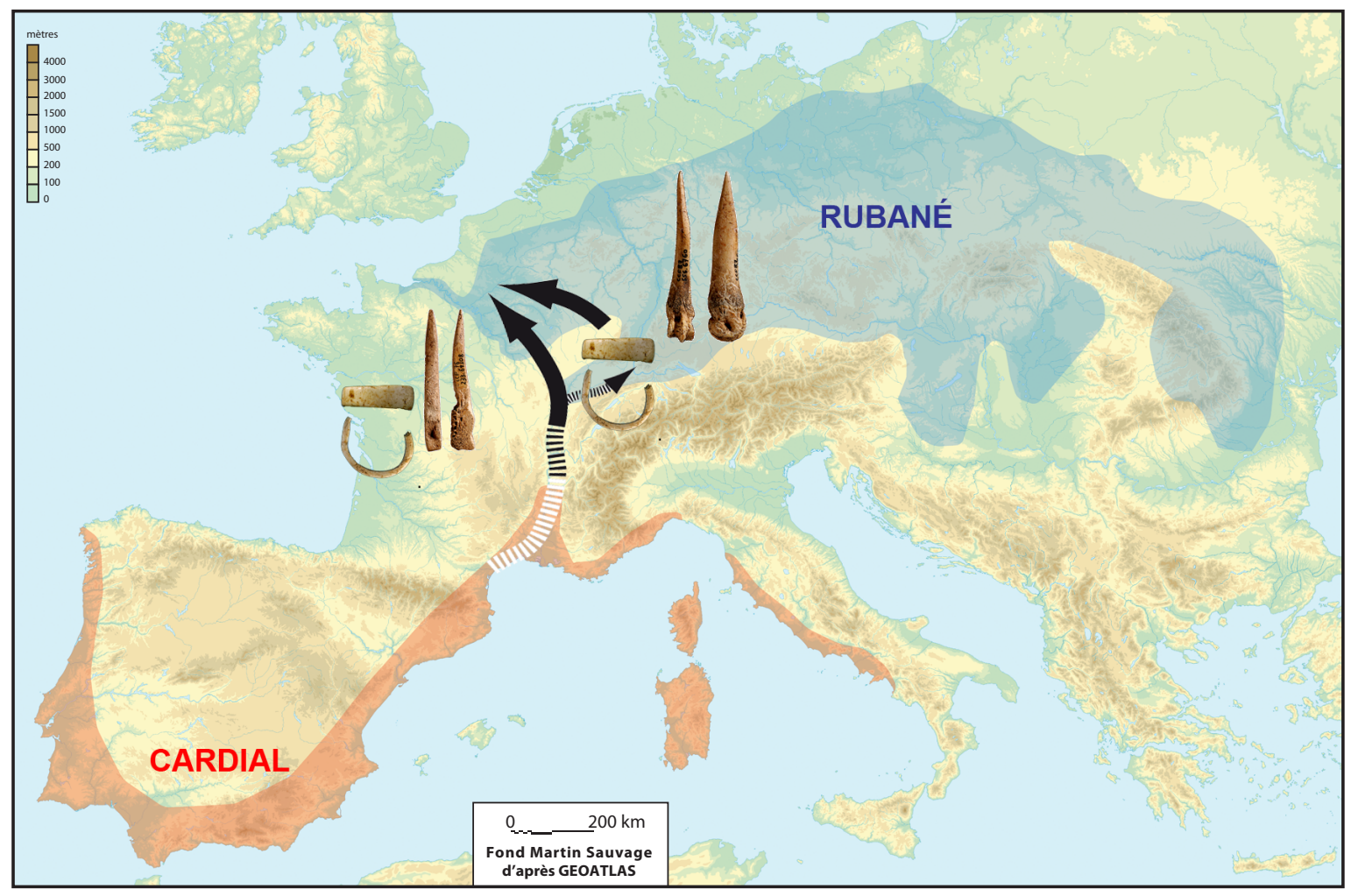


par exemple, à la Champagne. Bien entendu, ceci concernerait le déplacement de quelques individus, non pas de groupes entiers.

Si les productions osseuses des bâtiments comportent des signatures identitaires extra-régionales à l'intérieur d'un même site, alors elles mettent en avant l'aspect non homogène des occupants des maisons des villages au Rubané et donnent une perspective anthropologique nouvelle à la diffusion du Néolithique et à sa chronologie. L'installation de ces villages, contrairement à ce que l'on pensait jusqu'à présent, ne résulterait en définitive pas tant du déplacement d'une seule population, tels des colons venus d'un endroit et s'installant à un autre, mais peut être plutôt d'une agrégation d'individus, dont certains sont issus de différentes régions. Sur le principe de l'exogamie, ces déplacements pourraient correspondre à des réseaux d'échanges des femmes en même temps que de biens. Ainsi, sur le modèle des sociétés patrilinéaires sans classe de Nouvelle-Guinée, les Baruyas, M. Godelier (1984) fournit un motif concret au mariage et à la mobilité inter-communautaire des femmes. Selon lui, l'échange des femmes repose sur un réseau d'alliance nécessaire pour assurer la survie économique et la reproduction biologique et sociale des groupes, en raison de l'ingalité de la répartition des ressources (Godelier, 1984, p. 51). Ceci entraîne des rivalités et la mise en place de réseaux d'échanges dont font partie les femmes, selon une logique sociale de dons et de contre-dons et du principe de l'équivalence des femmes : une femme en vaut une autre (Godelier, 1984, p. 32-60).

Ces questions d'emprunts techniques et d'intermariages ont fait l'objet d'études ethno-archéologiques en particulier sur la céramique, avec pour objectif de comprendre comment l'origine d'un individu se concrétise dans la culture matérielle (Gosselin, 2002; Gelbert, 2003; Degoy, 2008). Toutes ces études montrent tout d'abord combien les alliances peuvent favoriser le déplacement des potières, et avec elles combien les pra- tiques techniques acquises au sein de leur communauté d'origine transforment tout en les intégrant celles de leur communauté d'arrivée. A. Gelbert, par exemple, met l'accent sur la fragmentation des emprunts techniques des potières déplacées à la tradition d'accueil (Gelbert, 2003, p. 47-50). Dans l'industrie osseuse rubanée, il n’y a cependant aucune fragmentation dans les savoir-faire, mais une reproduction sans interprétation des morphologies et des chaînes opératoires telles qu'à l'initial. En outre, les emprunts à la tradition cardiale (Cardial ou Épicardial) s'intègrent parfaitement sans transformation du fonds rubané. Ils ne compromettent en aucun cas l'équilibre du milieu technique pour citer Leroi-Gourhan (1973), ne faisant qu'ajouter des formes et des techniques nouvelles aux existantes.

Les signatures régionales que pourraient comporter la production osseuse des bâtiments rubanés serait dues aux variations des traditions de fabrication dont seraient issues les fabricantes. De fait, l'évolution de cet outillage ne peut être linéaire y compris dans des tranches chronologiques étroites comme entre les étapes du RRBP, qui embrassent une centaine d'années. Tenant de faits anthropologiques, elle se construit au contraire sur l'irrégularité et la rupture. Ceci pose, en tout cas, de nouveaux paradigmes pour le Néolithique rubané, que sont l'extrême mobilité des personnes, la perméabilité sociale des groupes et la variété de la composition populationnelle des villages. Ces pistes de recherche seront à exploiter en synergie avec les autres matériaux de la recherche rubanée.

\section{NOTE}

(1) L'information détaillée est actuellement disponible dans un travail universitaire (Sidéra, 2010), et sera accessible dans un volume à paraître de la publication monographique de Cuiry-lès-Chaudardes. Différents autres détails, auxquels le lecteur pourra se rapporter, viennent d'être publiés dans une monographie récente (Sidéra, 2012).

\section{RÉFÉRENCES BIBLIOGRAPHIQUES}

Allard P. (2005) - L'industrie lithique des populations rubanées du Nord-Est de la France et de la Belgique, Rahden, Marie Leidorf (Internationale Archäologie, 86), 280 p.

Bosch I Lloret A., Chinchilla Sanchez J., Tarrus I Galter J. (2000) - El poblat lacustre neolitic de la Draga. Excavacions de 1990 a 1998, Gérone, Museu d'arqueologia de Catalunya, Centre d'arqueologia subaquàtica de Catalunya, 296 p.

Constantin C., Ilett M. (1997) - Une étape finale dans le Rubané récent du Bassin parisien, in C. Jeunesse (dir.), Le Néolithique danubien et ses marges entre Rhin et Seine,

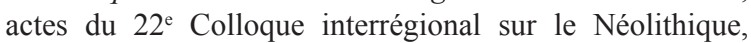
(Strasbourg, 27-29 octobre 1995), Strasbourg, Association pour la promotion de la recherche archéologique en Alsace (Monographies d'archéologie alsacienne, 3), p. 281-300.

Degoy L. (2005) - Variabilité technique et identité culturelle: un cas d'étude ethnoarchéologique en Andhra Pradesh (Inde $d u S u d)$, thèse de doctorat, université Paris X, Nanterre.
Fehlmann D. (2010) - Worked Bone, Tooth and Antler Objects from the Early Neolithic Site of Asparn/Zaya-Schletz (Lower Austria), in A. Legrand-Pineau, I. Sidéra, N. Buc, E. David, V. Scheinsohn, D. V. Campana, A. M. Choyke, P. Crabtree et A. Stone (dir.), Ancient and Modern Bone Artefacts from America to Russia. Cultural, Technological and Functional Signature, Oxford, Archaeopress (British Archaeological Reports, International series 2136), p. 31-40.

Fritsch B. (1998) - Die linearbandkeramische Siedlung Hilzingen „Forsterbahnried” und die altneolithische Besiedlung des Hegaus, Rahden, Verlag Marie Leidorf, 296 p.

Gallay G., Mathieu G. (1988) - Grabbeigaben der Bandkeramik von Ensisheim dep. Haut-Rhin (Elsass), Germania, 66, 2, p. 371-389.

Gelbert A. (2003) - Traditions céramiques et emprunts techniques dans la vallée du Fleuve Sénégal, Paris, Éd. de la MSH - Épistèmes (Référentiels, 1), 104 p. 
HAAсK F. (2012) - Die Knochen-, Zahn- und Geweihartefakte aus Herxheim, DFG-Projekt ,Siedlung und Grubenanlage Herxheim b. Landau" [en ligne : http://www.projekt-herxheim.de/knogeza.htm].

HÜSER A. (2002) - Bandkeramische Knochen-, Geweih- und Zahnartefakte aus Bad Nauheim-Nieder-Mörlen, Wetteraukreis, in E. Schallmayer (dir.), Hessen Archäologie 2001, Wiesbaden, Landesamt für Denkmalpflege Hessen, p. 32-34.

Godelier M. (1984) - L'idéel et le matériel. Pensée, économie, société, Paris, Fayard, 348 p.

Jeunesse C. (1995) - Les relations entre l'Alsace et le Bassin parisien au Néolithique ancien vues à travers l'étude des pratiques funéraires, in C. Billard et Lemercier M. (dir.) Actes du $20^{e}$ Colloque interrégional sur le Néolithique Evreux 1993, Rennes, Association pour la diffusion des recherches archéologiques dans l'Ouest de la France (Supplément à la Revue archéologique de l'Ouest, 7), p. 13-20.

KaufmanN D. (1989) - Kultische Äusserungen im Frühneolithikum des Elbe-Saale-Gebietes, in F. Schlette et D. Kaufmann (dir.), Religion und Kult in ur- und frühgeschichtlicher Zeit, Berlin, Akademie-Verlag (Auftrag der Historiker-Gesellschaft der DDR, 13), p. 111-140.

Leroi-Gourhan A. (1973) - Évolution et techniques, II. Milieux et techniques, Paris, Albin Michel, 475 p.

Mathieu G. (1983) - Une nouvelle station rubanée à Ensisheim, Bulletin de la Société préhistorique française, 80, 10-12, p. 349-359.

Poplin F. (1975) - La faune danubienne d'Armeau (Yonne, France) : ses données sur l'activité humaine, in A. T. Clason (dir.), Archaeozoological Studies, New York, Elsevier, p. 179-192.

Poplin F. (1976) - Étude comparative de deux séries de chasselames en bois de cerf néolithique de l'Yonne (France) et du Missouri (USA), Congrès préhistorique de France, $20^{e}$ session, 1974, Provence, Paris, Société préhistorique française, p. 499-505.

Poplin F. (1980) - Des chasse-lame néolithiques en bois de cerf de l'Yonne, de Spiennes et pourquoi pas du GrandPressigny, in Études sur le Néolithique de la région Centre, actes du $5^{\text {e }}$ Colloque interrégional sur le Néolithique (SaintAmand-Montrond, 1977), Saint-Amand-Montrond, Association des amis du musée Saint-Vic, p. 41-48.

Poulain T. (1983) - Note sur l'outillage osseux, Bulletin de la Société préhistorique française, 80, 10-12, p. 360-362.

RücK O. (2001) - Eine bandkeramische Siedlung bei Utzwingen, Gemeinde Maihingen, Landkreis Donau-Ries, Schwaben, Das archäologische Jahr in Bayern 2000, p. 17-19.

RulF J. (1984) - Příspěvek k poznání neolitické kostěné industrie v Cechách [A Contribution to the Study of Neolithic Bone Industry of Bohemia], Archeologické rozhledy, 36, 3, p. 241-260.

Schliz A. (1901) - Das steinzeitliche Dorf Grossgartach, seine Kultur und die spätere vorgeschichtliche Besiedlung der Gegend, Stuttgart, Enke, 52 p.

SIDÉRA I. (1989) - Un complément des données sur les sociétés rubanées : l'industrie osseuse à Cuiry-lès-Chaudardes, Oxford, Archaeopress (British Archaeological Reports, International Series 520), 208 p.

SIDÉRA I. (1993) - Les assemblages osseux en bassins parisien et rhénan du VIe au IVe millénaire BC. Histoire, techno-éco- nomie et culture, thèse de doctorat, université Paris 1 - Panthéon-Sorbonne, $636 \mathrm{p}$.

SidÉRA I. (1998) - Die knochen-, Geweih- und Zahnartefakte aus Vaihingen - Ein Überblick, in R. Krause (dir.), Die bandkeramischen Siedlungsgrabungen bei Vaihingen an der Enz, Kreis Ludwigsburg (Baden-Würtemberg), Bericht der Römisch-Germanischen Kommission, 79, p. 81-92.

SidÉRA I. (2000) - Animaux domestiques, bêtes sauvages et objets en matières animales du Rubané au Michelsberg. De l'économie aux symboles, des techniques à la culture, Gallia Préhistoire, 42, p. 108-194.

SidÉra I. (2008) - Rubané, VSG et Cardial. Filiations de l'industrie osseuse, in L. Burnez-Lanotte, M. Ilett, et P. Allard (dir.), Fin des traditions danubiennes dans le Néolithique du Bassin parisien et de la Belgique (5100-4700 av. J.-C), Paris, Société préhistorique française (Mémoire, 44), p. 209-219.

SIDÉRA I. (2010) - Cuiry-lès-Chaudardes. De l'analyse technique et fonctionnelle de l'industrie osseuse de à la perception de réseaux de parenté, thèse d'habilitation à la direction de recherches, université Paris-Ouest - NanterreLa Défense [à paraître dans la série de monographies sur Cuiry-lès-Chaudardes, Rahden, Marie Leidorf (Internationale Archäologie)].

Sidéra I., avec la collaboration de Barthélémy de SAISIEUX B., JabBour-SAVAlle D. (2010) - Des migrants d'origine méditerranéenne dans le Bassin parisien au Néolithique ancien : technologie et morphologie de l'industrie osseuse, The Arkeotek Journal, 3, 2 [en ligne : www.thearkeotekjournal.org].

SIDÉRA I. (2012) - Nouveau regard sur la néolithisation. L'industrie osseuse de l'Anatolie au Bassin parisien via la Méditerranée, Paris, de Boccard (Travaux de la Maison René-Ginouvès, 15), 106 p.

SpATz H. (1988) - Bemerkungen zu den Artefakten aus Knochen und Geweih, in H. Spatz, S. Alföldy-Thomas (dir.), Die „Grosse Grube” der Rössener Kultur in Heidelberg-Neuenheim, Stuttgart, Konrad Theiss (Materialhefte zur Vor- und Frühgeschichte in Baden-Württemberg, 11), p. 28-53.

Werning J.A. (1983) - Die Geweihartefakte der neolitischen Moorsiedlung Hüde I am Dümmer, Kreis Grafschaft Diepholz, Neue Ausgrabungen und Forschungen in Niedersachsen, 16, p. 21-187.

Yravedra Sainz De Los Terreros J., Maicas Ramos R., Consuegra Rodriguez S., Diaz Del Rio Español P. (2008) - Anillos para una mina, industria osea y fauna de la mina de silex néolítica de Casa Montero (Madrid), in M. S. Hernández Pérez, J. A. Soler Díaz et J. A. López Padilla (dir.), IV Congreso del Neolitico Peninsular, actes du congrès (Alicante, 27-30 novembre 2006), vol. 2, Alicante, Museo Arqueologico de Alicante, p. 240-247.

Isabelle SIDÉRA

UMR 7055 «Préhistoire et technologie » Maison de l'archéologie et de l'ethologie 21, allée de l'Université 92023 Nanterre cedex isabelle.sidera@mae.u-paris10.fr 